\title{
Complex Magnetic Anomalous Chaotic Inversion
}

\author{
Jiaxiong Cai ${ }^{1}$, Yueping Yan² \\ ${ }^{1}$ Hunan Provincial Department of Natural Resources, Changsha, China \\ ${ }^{2}$ Team 247 of Hunan Provincial Nonferrous Metals Geological Exploration Bureau, Changsha, China \\ Email: 827900498@qq.com
}

How to cite this paper: Cai, J.X. and Yan, Y.P. (2021) Complex Magnetic Anomalous Chaotic Inversion. International Journal of Geosciences, 12, 1071-1083.

https://doi.org/10.4236/ijg.2021.1212057

Received: October 27, 2021

Accepted: December 20, 2021

Published: December 23, 2021

Copyright $\odot 2021$ by author(s) and Scientific Research Publishing Inc. This work is licensed under the Creative Commons Attribution International License (CC BY 4.0). http://creativecommons.org/licenses/by/4.0/

\begin{abstract}
In the complex magnetic anomalies under undulating terrain, multiple source superposition and significant noise conditions, the "noise avoidance inversion" depends on three experiments. The displacement experiment accurately solves the prediction problem of the form of the target magnetic source, finds the matching relationship with the maximum thickness of the target magnetic source, and the noise change experiment demonstrates the reliability of the noise avoidance inversion. It can be seen that "noise avoidance inversion" creates a "chaotic image back to true" inversion route for complex magnetic anomalies. Noise avoidance inversion of multi-source superimposed complex magnetic anomalies in Erhai Lake is an example.
\end{abstract}

\section{Keywords}

Variable Model, Permutation Experiment, Solidification Noise, Recognition Function, Morphological Discrimination, Noise Avoidance Inversion

\section{Prologue}

In the topic of complex magnetic anomaly, it is difficult to predict the magnetic source morphology of the target anomalies, and then comprehensively explain the abnormalities in the inversion field of complex magnetic anomalies. This is difficult to mention, should be the wish of many scholars.

\section{Theoretical [1]-[11]}

Studying the target anomalies in avoidance noise inversion in complex abnormalities seems an inconceivable puzzle. However, when a "recognition function" can identify the abnormal magnetic source form of the target on the spot, the bottleneck problem of solving the problem will make substantial progress. This is the original idea of "noise avoidance reaction". 
Variable model is the model basis of noise avoidance inversion; elevation tracking is the method support of noise avoidance inversion; remutation experiment is the experimental basis of noise avoidance inversion discovery, "recognition function" and noise avoidance.

\subsection{Variable Model [12] [13]}

Theoretically, variable models can be equivalent to any magnet model of independent morphology and produce anomalies with unique features on arbitrary undulating topography. Importantly, the following formula can easily give three models of the thickening of the magnet, the book, the unchanged tip body, the lens body, and the plate body. Under the rolling terrain conditions, the magnetic field expression of lens shape, plate body, pointed extinguishing body and other forms can be written as:

$$
\begin{gathered}
\Delta T_{x y}=\frac{1}{n} \sum_{i=1}^{n} 2 b_{i} J_{S} \sin \beta \sqrt{1-\cos ^{2} I_{0} \cos ^{2} A} F_{i}\left(h_{x y} x, y \pm L_{i}, l_{i}\right) \\
F_{i}\left(h_{x y}, x, y \pm L_{i}, l_{i}\right)=f\left(h_{x y}, x, y+L_{i}, l_{i}\right)-f\left(h_{x y}, x, y-L_{i}, l_{i}\right) \\
f\left(h_{x y}, x, y+L\right)=\frac{L_{z}}{\sqrt{c^{2}+L_{z}^{2}}} \frac{(x-l \cos \beta) \cos \xi-\left(h_{x y}+l \sin \beta\right) \cos \xi}{(x-l \cos \beta)^{2}+\left(h_{x y}+l \sin \beta\right)^{2}} \\
\left.-\frac{L_{z}\left(x \sin \xi-h_{x y} \cos \xi\right)}{\sqrt{\left(D^{2}+L^{2}\right)}-\frac{1}{D^{2}}\left(\frac{P+1}{B^{2}+L_{z}^{2}}\right)}-\frac{P}{\sqrt{c^{2}+L_{z}^{2}}}\right)((\cos \xi \cos \beta \\
\left.\left.-\cos \xi-\cos ^{2} \xi+K^{2}\right) L_{z}-2 k \sin \left(i_{s}-\beta\right) B\right) \\
+2 K \cos \left(i_{s}-\beta\right)\left(\frac{1}{\sqrt{c^{2}+L_{z}^{2}}}-\frac{1}{\sqrt{D^{2}+L_{z}^{2}}}\right)
\end{gathered}
$$

where: $L_{z}=Y+L ; L_{f}=Y-L ; P=h_{x y} \sin \beta-x \cos \beta ; B=x \sin \beta+h_{x y} \cos \beta$; $D^{2}=x^{2}+h_{x y}^{2} ; c^{2}=(x-l \cos \beta)^{2}+\left(h_{x y}+l \sin \beta\right)^{2} ; K=\cos i_{s} \operatorname{ctg} A ; \quad \xi=2 i_{s}-\beta ;$ $i_{s}=\operatorname{arctg}\left(\operatorname{tg} I_{0} \csc A\right) ; h_{x y}=h+H(x, y) ; b_{i}=b_{1}, b_{2}, b_{3}, \cdots, b_{n}$; $L_{i}=L_{1}, L_{2}, L_{3}, \cdots, L_{n} ; l_{i}=l_{1}, l_{2}, l_{3}, \cdots, l_{n} ; H(x, y)$ for height data with zero origin; $J_{s}$, effective magnetization within the profile; $\xi$, magnetization feature angle; $L$, half length of the direction of the magnetic source; 1 , Magnetic source depth elongation; $b$, half thickness of magnetic source; $\beta$, magnetic source inclination; $I_{0}$, geomagnetic inclination; $i_{s}$, effective magnetization inclination within the profile; $A$, magnetic source azimuth.

Using the above variable model formula, different sizes of book plates are selected, parallel superimposed, constructed in the profile: the "pointed body with thick top and narrow bottom"; narrow "lens body" up to the bottom and thick in the middle; up and down equally thick "thick plate body". The three types of models are the basis of forming the "model three methods" to identify magnet morphology.

"Model three methods" refers to the combination of methods using the exclusive interpretation method of three models to identify the same target abnormal- 
ity in complex abnormalities.

\subsection{Elevation Tracking [14] [15]}

Elevation tracking is to use the theoretical abnormalities with the terrain influence to explain the field abnormalities affected by the terrain, therefore, for the terrain impact, no need to handle, but the use, is also resolved.

Elevation tracking, "tracking" refers to the magnetic source with different buried depths in the profile. It can automatically use the elevation of the surface projection on the magnet as zero, and take the buried depth scale as the "relative elevation" explaining the corresponding magnetic source anomalies, which can virtually resolve the impact of terrain on the anomalous inversion process.

Elevation tracking formula for resolving the terrain influence for noise avoidance and inversion:

$$
H_{m}(i)=H(i)-H\left(I N T \frac{N}{2}+J J\right)
$$

In type, $H_{m}(i)$, elevation tracking array; $H(i)$, section elevation array; $H\left(I N T \frac{N}{2}+J J\right)$, exception origin elevation; $J J$, number of points where the origin deviates from the point in the profile.

\subsection{Replacement Experiment}

For the three replacement experimental sections, plate abnormal interpretation method, lens abnormal interpretation method (model three methods) were used, and three groups and nine interpretation results were obtained.

\subsubsection{The Recognition Function}

Using the integration difference of magnetic moment and outlier absolute value of nine interpretation results in the front and after three groups, through alignment, screening and combination, and finally found an "identification function" that can correctly distinguish the abnormal magnetic source after the target anomalous magnetic source morphology is replaced $\varphi$.

$$
\varphi=\frac{\Delta T_{00}-\sqrt{\Delta T_{S Y}}}{\Delta T_{00}+\Delta T_{D J}} \sqrt[3]{\frac{M_{S}}{M_{B}}}
$$

$\Delta T_{00}$, integrvalues of complex outliers; $\Delta T_{S Y}$, the remaining outliers are absolute integral value; $\Delta T_{D J}$, superimposed outliers absolute values; $M_{S}$, the model method explains the magnetic moments in the target anomaly results; $M_{B}$, the bookkeeping method explains the magnetic moment results of the target exception.

The Recognition Parameter consists of two information: curve ratio and magnetic moment ratio.

Curve ratio refers to the three types of curves obtained by the model three-way method, where the superposition anomalies involved include the superimposed anomalies prior to the interpretation of the target anomalies. The remaining ano- 
malies refer to the difference between the complex outlier absolute integral value and the superimposed outlier absolute integral value

Magnetic moment ratio refers to the magnetic moment ratio obtained by model 3 method and accounting interpretation method explaining target anomalies respectively.

The recognition function interpretation of the structure is the reverse difference between the curve and the magnetic moment of the three model methods to explain the target anomaly. Only the recognition function of this combination is chosen to directly discriminate the anomalous magnetic source morphology of the target.

The results in Table 1, combined with the analysis of Figure 1, show that the overall characteristics of abnormalities in the three substitution experimental profiles are only weakly different. Using identification parameters to identify the classification information of magnetic source form must have two preconditions: 1) The factors (noise) affecting the interpretation method of the three types of models are unchanged; 2) The identification parameters must be suitable for the classification of substitution abnormalities in the three models with high accuracy. Table 1 results demonstrate that the two necessary premises do exist.

Table 1. Replacement experiment results.

\begin{tabular}{|c|c|c|c|}
\hline $\begin{array}{l}\text { Replacement } \\
\text { experiments }\end{array}$ & recognition methods & $\begin{array}{c}\text { Identify } \\
\text { parameters }\end{array}$ & $\begin{array}{l}\text { Identify } \\
\text { results }\end{array}$ \\
\hline Abnormal thick & Abnormal thick plate interpretation & 23.85 & \multirow{3}{*}{$\begin{array}{c}\text { Abnormal } \\
\text { thickness } \\
\text { plate }\end{array}$} \\
\hline $\begin{array}{l}\text { plate body } \\
\text { replacement }\end{array}$ & Lens body abnormal interpretation & 22.98 & \\
\hline "D" abnormal & Interpretation method of cone & 22.10 & \\
\hline Lens body & Abnormal thick plate interpretation & 6.79 & \multirow{3}{*}{$\begin{array}{l}\text { Lens body } \\
\text { abnormal }\end{array}$} \\
\hline $\begin{array}{l}\text { abnormal } \\
\text { replacement }\end{array}$ & Lens body abnormal interpretation & 17.03 & \\
\hline "D" abnormal & Interpretation method of cone & 9.52 & \\
\hline Conical & Abnormal thick plate interpretation & 22.84 & \multirow{3}{*}{$\begin{array}{c}\text { Conical } \\
\text { abnormalities }\end{array}$} \\
\hline $\begin{array}{c}\text { abnormalities } \\
\text { replacement }\end{array}$ & Lens body abnormal interpretation & 20.41 & \\
\hline "D" abnormal & Interpretation method of cone & 25.5 & \\
\hline
\end{tabular}

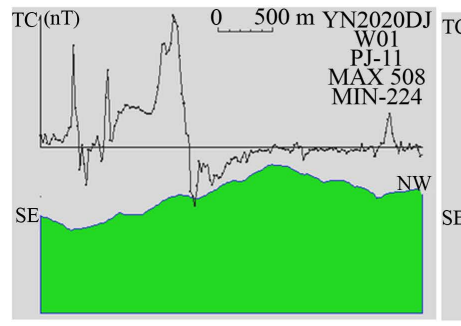

(a)

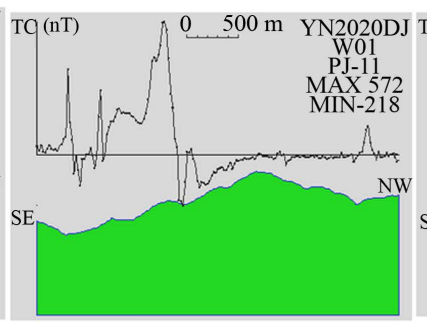

(b)

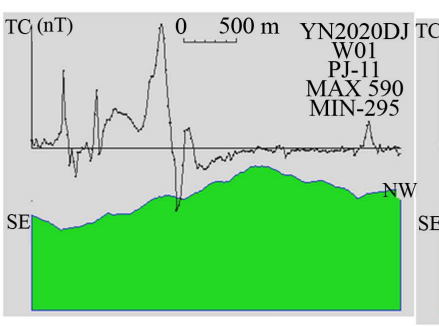

(c)

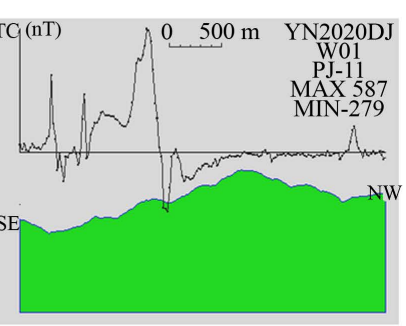

(d)

Figure 1. Section of the displacement experiment. Figure Note a shows the original profile of magnetic abnormal W01 in Erhai Lake; b, c, d is the profile of the abnormal replacement target abnormality of thick plate body, lens body and pointed elimination body model, respectively. 
Factors affecting the three types of model interpretation methods, including topographic influence, superposition effects of anomalies, and high-frequency interference. When these effects explain the substitution anomaly by using the model three method, they are the invariant state of instantaneous "fixed frame", which is called "curing noise", and the test of the model three method does not mask the characteristic information of the magnetic source. It is therefore possible to be revealed and distinguished by the recognition function. It is reasonable to argue that the replacement experiment demonstrates the "curing noise".

At this point, the replacement experiment finally found the way to determine the attribution of the magnetic source category by relying on the model threemethod plus recognition function to determine the target anomalies in the measured abnormality.

After the "model three-method-recognition function" solves the target anomalous magnetic source morphology, the interpretation process of complex magnetic anomalies can be completed objectively, and we give close to the actual interpretation results. This is the theoretical basis for noise avoidance inversion to solve the bottleneck problem.

Model three-method-recognition function tests the " $D$ " anomaly in W01, whose field source morphology is pointed body, and the deep source anomaly of G" is lens.

The displacement experiment gives an effective method to identify the morphology of target abnormal magnetic sources in complex abnormalities: "model three methods-parameter identification". It can be seen that the "replacement experiment" is the experimental theoretical basis for the noise avoidance inversion.

\subsubsection{Judge Thick Experiment}

Under the premise of the target abnormal morphology, the complex abnormal inversion process is suspended. At that time, the thickness can be adjusted to observe the changes of the remaining abnormal, and the thickness of the remaining abnormal minimum point is, the maximum thickness of the target abnormal magnetic source. The principle is that the remaining anomaly is only minimized when the magnetic source morphology of the target anomaly is consistent with the thickness.

The so-called noise that explains the target anomalies, includes many factors. During topographic effects, the effects of adjacent abnormal errors, the effects of remaining superimposed anomalies in residual abnormalities that have not been explained, and the high-frequency interference in the presence of shallow layers. These effects are completely different in different stages of the interpretation of complex anomaly profiles, but are "fixed", and transiently invariant "cured noise".

W01 explained section " $\mathrm{D}$ " abnormal morphology, identified as pointed body, the maximum thickness of the top is $92 \mathrm{~m}$ by thickness (see Table 2). 
Table 2. Erhai magnetic anomalous W01 section "D" target abnormal magnetic source thickness determination.

\begin{tabular}{ccccc}
\hline $\begin{array}{c}\text { Try the } \\
\text { thickness } \\
\mathrm{m}\end{array}$ & $\begin{array}{c}\text { Residual } \\
\text { outliers } \\
\mathrm{nT}\end{array}$ & $\begin{array}{c}\text { Identify } \\
\text { parameters }\end{array}$ & $\begin{array}{c}\text { Determine } \\
\text { thickness results } \\
\mathrm{m}\end{array}$ & $\begin{array}{c}\text { Relative } \\
\text { error } \\
\%\end{array}$ \\
\hline 20 & 7204.05 & 20.8726 & & \\
40 & 7192.11 & 20.9911 & & \\
80 & 7142.50 & 21.4822 & & \\
90 & 7124.26 & 21.6626 & & \\
92 & 7079.21 & 21.9133 & & \\
94 & 7108.05 & 21.9111 & & \\
100 & 7102.51 & 21.8814 & & \\
110 & 7104.79 & 21.8806 & & \\
\hline
\end{tabular}

The model experiment found an identification function that directly distinguishes the magnetic source morphology attribution of target anomalies in complex anomalies, and gave effective methods to determine the magnetic source thickness.

Model trimethod, used to test target exception in W01 profile "D", results show that the magnetic source form in the W01 section is the pointed body, through the thickness of the maximum thickness of the pointed body is 92 meters. In the expanded application of deep source anomaly, the results of deep source anomaly "The magnetic source form of G" is the lens body. Thickness is $75 \mathrm{~m}$.

\subsubsection{Variant Noise Experiment}

Interference noise of different orders is integrated into complex anomalies to test whether it affects the determination of the magnetic source thickness to measure the varying noise. Whether in noise avoidance inversion, noise can still be considered as a constant "cured noise" without affecting the inversion result.

The comparison Figure 2 below shows the injection of different levels of high-frequency interference factors into complex anomalies, with the consistent "thickness interpretation results of D" obtained under strict judgment conditions.

During the process of magnetic source thickness determination, the noise interference is large or small and both transient and does not affect the thickness determination result. Doudoubled noise is incorporated into complex anomalies and its thickness determination does not change. See Table 3.

It can be seen that the "displacement experiment" utilizes a systematic experiment.

A theoretical and methodological basis for the noise avoidance inversion is provided. 


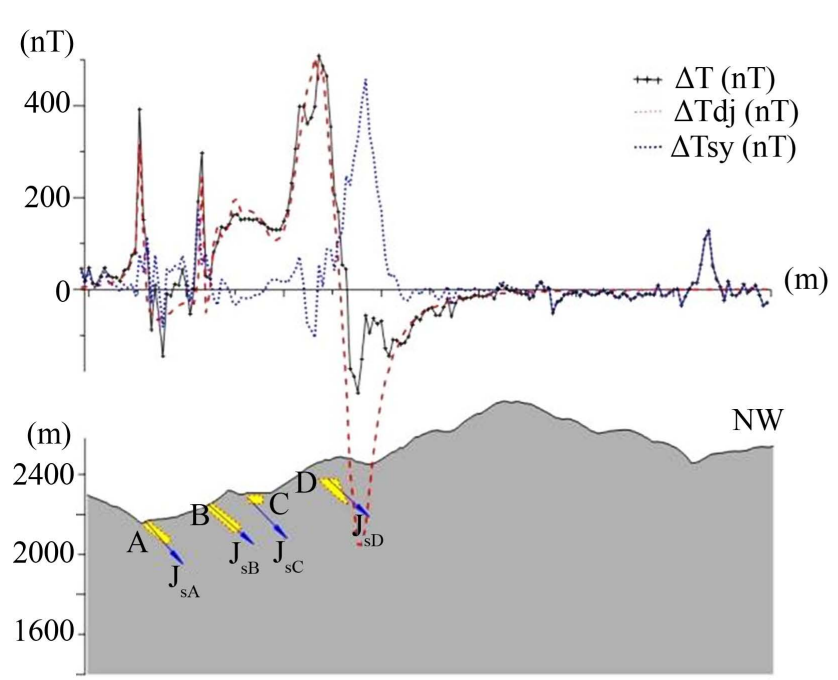

(a)

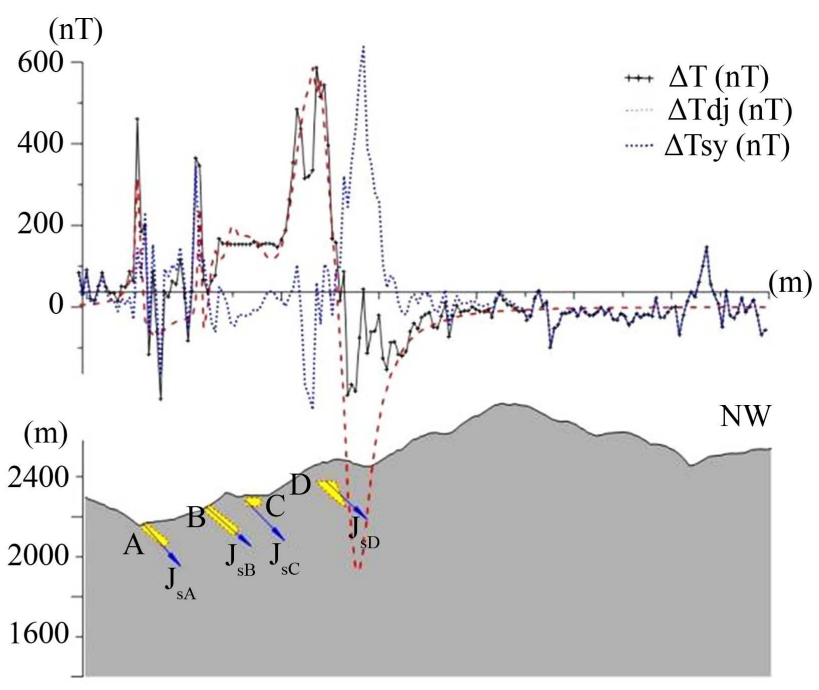

(b)

Figure 2. Injecting different shallow interference factors does not affect the thickness of the target magnetic source determined by noise avoidance inversion.

Table 3. The "D" magnetic source thickness determination table after noise doubling.

\begin{tabular}{ccccc}
\hline $\begin{array}{c}\text { Try the } \\
\text { thickness } \\
\mathrm{m}\end{array}$ & $\begin{array}{c}\text { Residual outliers } \\
\mathrm{nT}\end{array}$ & $\begin{array}{c}\text { Recognition } \\
\text { function }\end{array}$ & $\begin{array}{c}\text { Determine } \\
\text { thickness results } \\
\mathrm{m}\end{array}$ & $\begin{array}{c}\text { Relative } \\
\text { error } \\
\%\end{array}$ \\
\hline 20 & 10941 & 0.18161 & & \\
40 & 10988 & 0.19672 & \\
80 & 10930 & 0.27534 & \\
90 & 10924 & 0.32121 & \\
92 & 10921 & 0.34851 & \\
94 & 11006 & 0.34534 & \\
100 & 10922 & 0.34113 & \\
110 & 10937 & 0.21438 & \\
\hline
\end{tabular}

As shown from Table 3, when the trial thickness of the model varies greatly, both the remaining outliers and the recognition parameter values are small. Therefore, the credibility of controlling changes is particularly important. It was found that only if the maximum of the explanatory curve with varying thickness perfectly matched the maximum of the target anomaly. These two parameters are the most reliable.

Importantly, the thickness determination process also demonstrates the complex multi-element noise and does not mask the weak magnetic source thickness information; it demonstrates that the noise avoidance inversion avoids the noise effects and gives reliable inversion results. 


\section{Key Points of Noise Avoidance and Inversion}

Noise avoidance inversion was completed by three stages: "bookboard fitting", "morphological inversion" and "geological interpretation".

\subsection{Book Board Fitting}

It was found that independent morphological magnet anomalies with certain buried depth are not significantly different from the profile morphology of the book board anomaly, therefore, the book board anomalies are unified to simulate complex magnetic anomalies.

Under the premise of known latitude and longitude and determination of magnetic inclination angle, the abnormal fitting process can be easily completed according to the magnetic field decomposition, superposition and fitting principle, origin position, magnetic moment size, magnetic source depth, magnet inclination angle, length and delay change (see Figure 3). The initial interpretation results obtained by blackboard fitting are an important basis for "model interpretation".

\subsection{Optimize and Explain}

Its task: to identify the abnormal magnetic source form of the target through the "identification parameters", and on the basis of determining the thickness, supplemented by necessary adjustment, and strive to keep the form and thickness of the target abnormal magnetic source close to the actual state, to provide a reliable geophysical foundation for geological analysis.

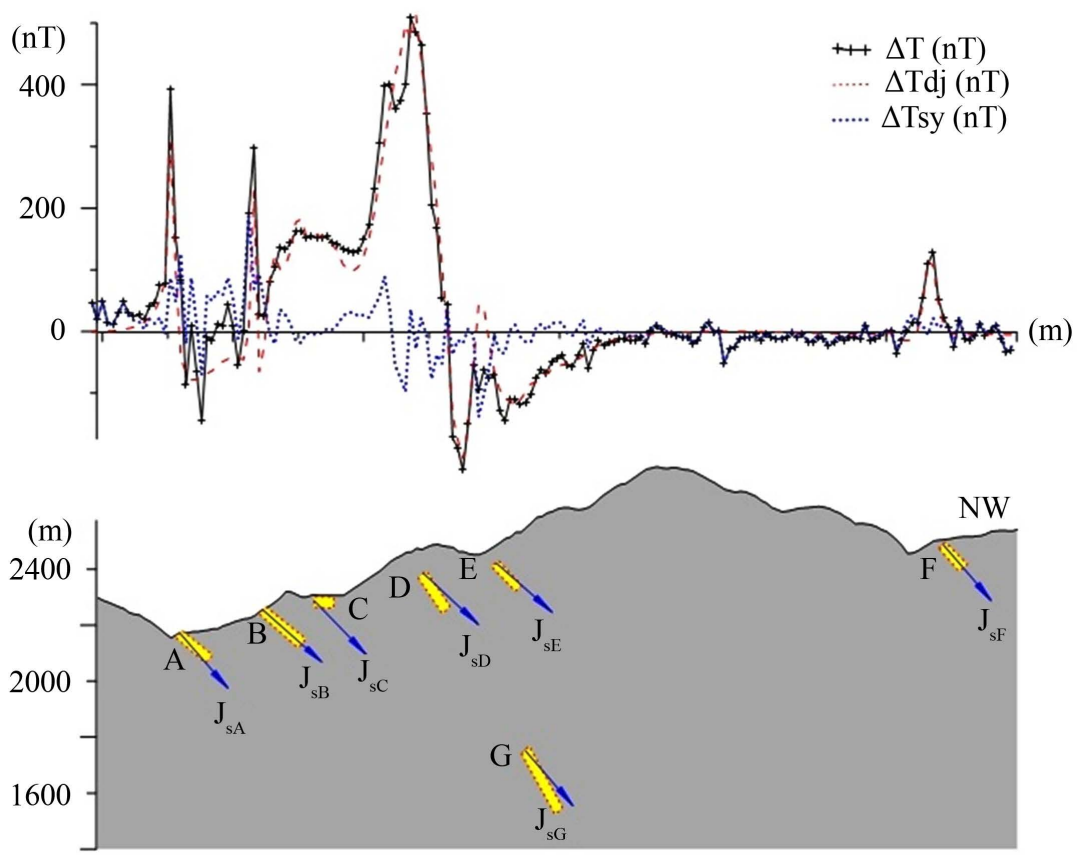

Figure 3. "Bookboard fitting" W01 complex magnetic anomaly section. $\Delta \mathrm{T}$ measured magnetic anomalies; $\Delta \mathrm{Tdj}$ overasing calculated magnetic exception; $\Delta \mathrm{Tsy}$ residual magnetic anomalies. 
The optimized interpretation shows that in the profile, the " $\mathrm{D}$ " anomalous magnetic source is the pointed body, the upper end is $92 \mathrm{~m}$ thick, and the magnetic source of the " $G$ " anomaly is the lens body, with an intermediate thickness of $70 \mathrm{~m}$.

Magnet data explaining the model anomalies, except for the thickness data, came from the bookkeeping fitting phase and were slightly modified if necessary (Figure 4).

\subsection{Geologic Interpretation}

Combined with the existing geological data, the morphological inversion results are used to draw the "geological interpretation profile of noise avoidance inversion". Verification recommendations are also made (Figure 5).

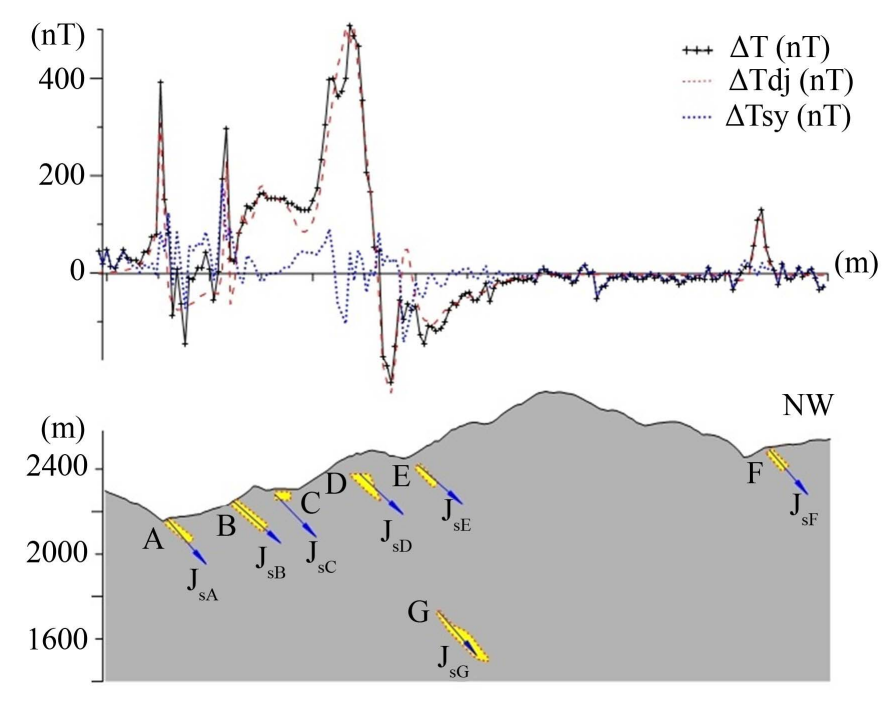

Figure 4. "morphological inversion" W01 complex magnetic anomaly profile.

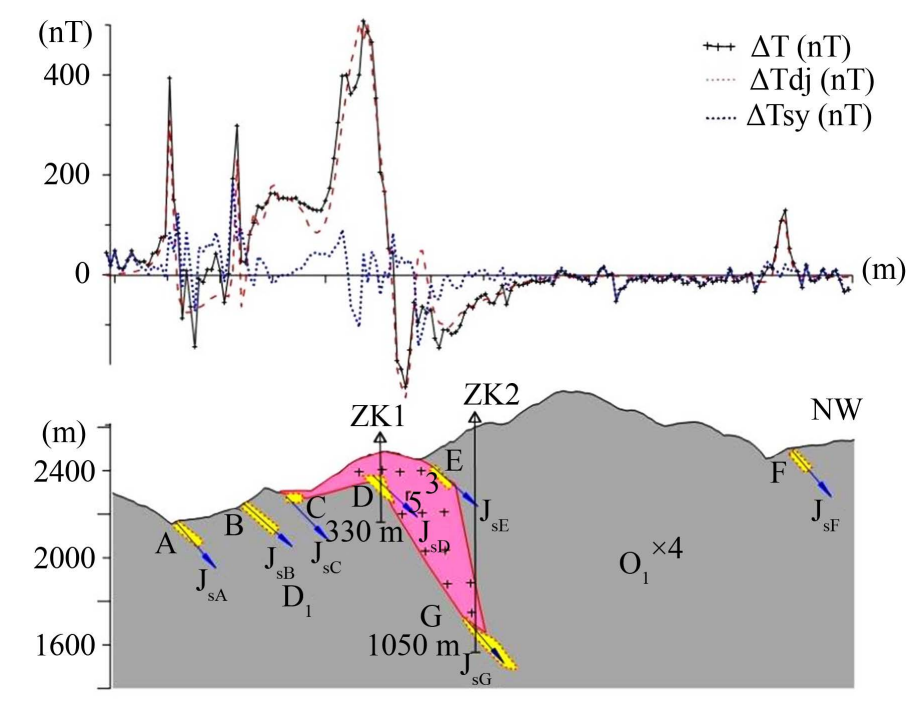

Figure 5. Geological interpretation section of magnetic anomalous noise avoidance in version of Erhai W01. 


\section{Instance Key}

Erhai magnetic anomaly is located at the foot of the Cangshan racetrack, undulating terrain. This anomaly is superimposed by multiple magnetic sources with significant high-frequency interference, and is a typical complex magnetic anomaly (Figure 6). Solve "heterogeneous platform problem"; hidden "E" abnormality is found; and deep source "G" abnormality with low frequency change. The inversion of the W01 complex anomaly was systematically performed using a "noise avoidance inversion".

\subsection{Ecverse Interpretation [16]}

In the same interpretation section, the abnormalities of different directions have different effective magnetization directions in the section, so they need to be handled differently.

The profiles with two abnormal reverse complexes from north to east show unique platform features. Therefore, in the process of book board fitting, its abnormal direction selection, we must make objective different from other target abnormalities. Through the heterogeneous treatment, the effective magnetization inclination in the profile and the effective magnetization inclination of the adjacent magnetic source. Ectropic treatment results: The abnormal platform features were well fitted (Figure 7).

\subsection{Depth Decomposition}

In the original superposition anomalies of W01, the "D", "E" anomalies are superimposed as similar to monomer anomalies etc. The absolute value of the strong negative value of the exception " $\mathrm{D}$ " is greater than the positive, releasing the "E" exception it overrides. To determine its authenticity, the corresponding "underwater peaks" (relative peaks in the "negative region" are easily found in the negative region below the "anomalous" peaks (Figure 8).

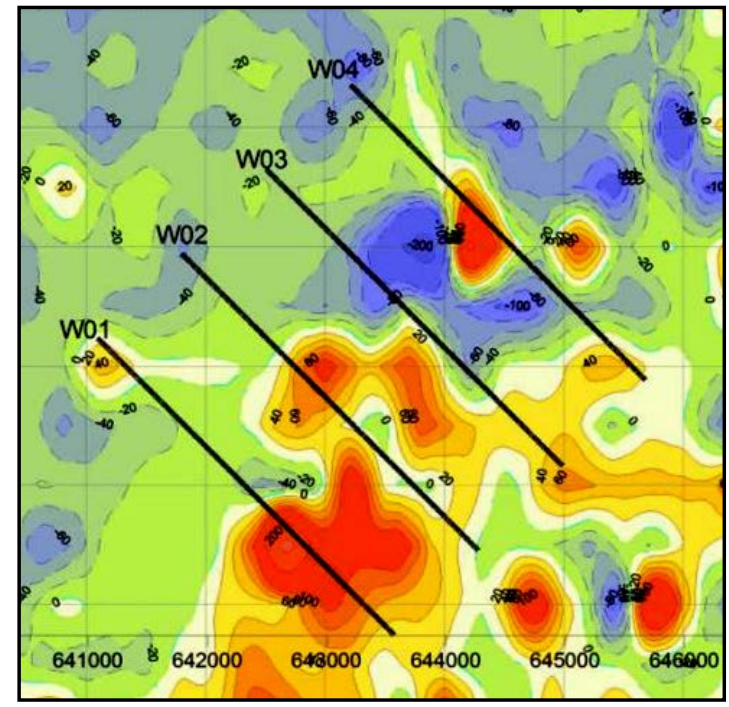

Figure 6. Magnetic anomaly Plan of Erhai Lake. 


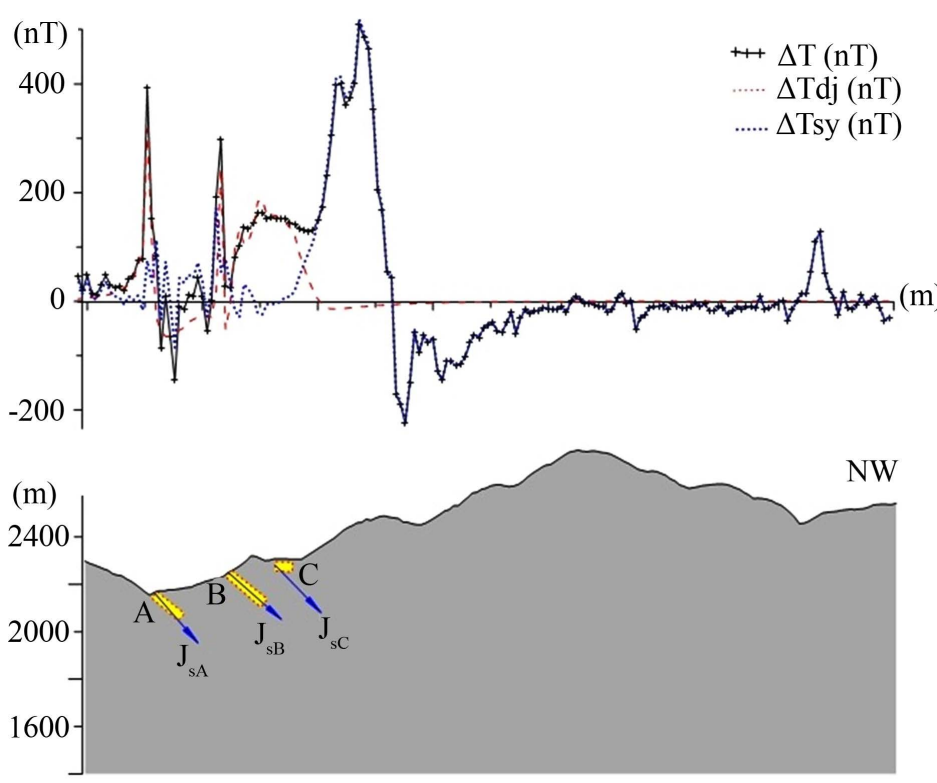

Figure 7. A heterotropic explanation of the "platform features of the C" anomalies.

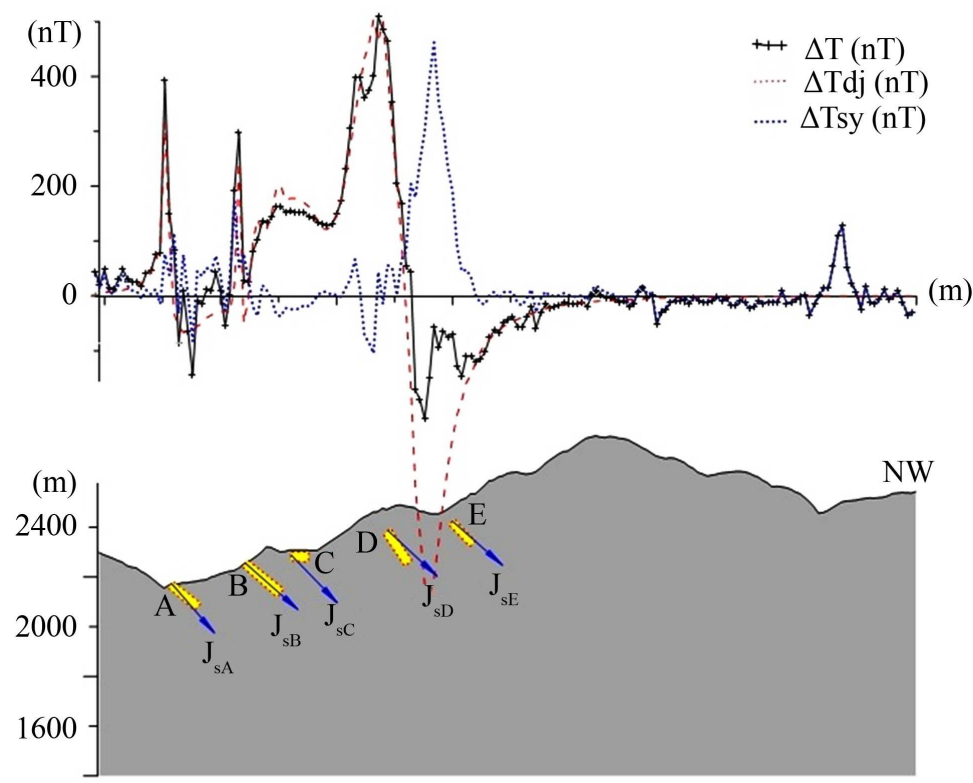

Figure 8. The masked "E" was found.

\subsection{The Deep Magnetic Source Is Revealed}

When the anomaly explains that "a, B, C, D, E" reaches "F", the "residual anomaly" presents a slow positive and negative low frequency feature, which is clearly important information for the presence of a deep source "G" (Figure 9). This should be considered an important finding.

\subsection{A Superficial Understanding}

The magnetic measurement data noise avoidance inversion results combined with the good prospecting prospect. 


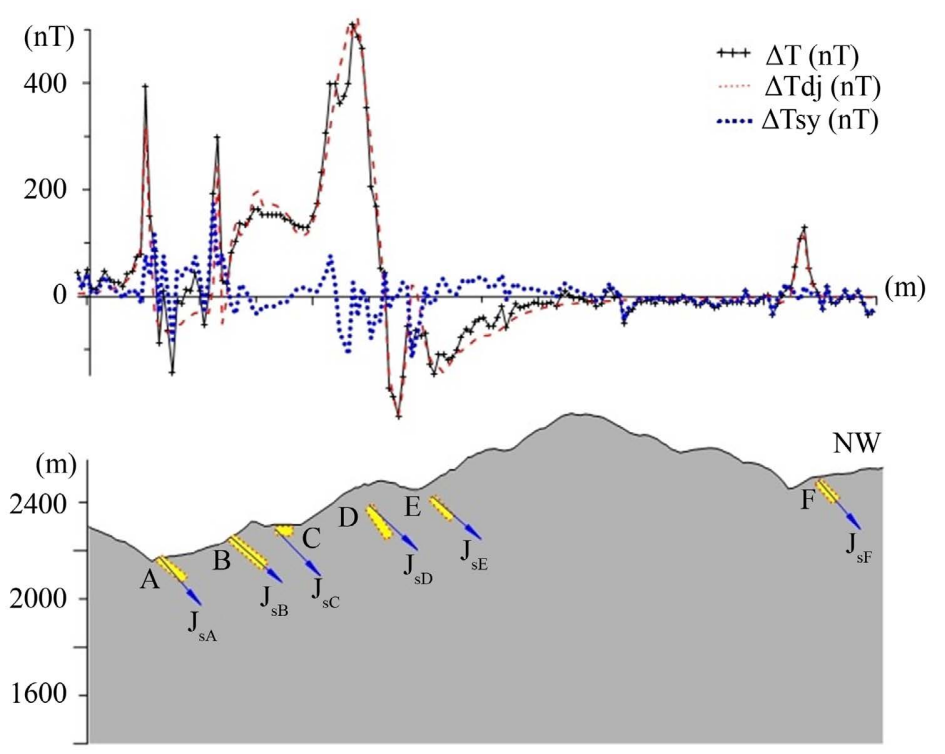

Figure 9. Deep Source anomaly “G” is revealed.

The complex morphology should be related to structure and hydrothermal activity after sub-magmatic phase.

The " $D$ " magnet, located at the deep extension bend of the rock body, is a larger so-called pointed fire extinguisher, which is suspected to be an "iron cap". Coincidentally, in the depths of the rock, there is a deep magnet " $G$ ". Between the two, although there are no magnets, this magnetic-free space, is indeed the most advantageous space to explore medium-heat polymetallic sediments.

\section{Conclusions}

The innovative way of "chaos inversion" comes from the establishment and demonstration of the new concept of "curing noise"; relies on the identification function of the target magnetic source morphology and thickness in complex magnetic anomalies; and finally develops the three-step combination of "noise avoidance inversion" through the model three method.

The "three methods" of the variable model is the method of noise avoidance inversion; the "identification parameter" obtained by the replacement experiment is the experimental theoretical basis of "noise avoidance inversion".

Hidden by many shallow magnet anomalies, buried nearly kilometer deep source abnormality can be found; successfully decompose complex anomalies with overlapping conditions and different directions, and complete the full parameter inversion respectively, which fully shows that noise avoidance inversion has important practical and generalized value.

Although the practice of noise avoidance inversion is insufficient, the advanced idea of simplifying complicated is worth continuing to deepen research.

\section{Conflicts of Interest}

The authors declare no conflicts of interest regarding the publication of this paper. 


\section{References}

[1] Gravity and Magnetic Methods (1965) The Quantitative Interpretation of Magnetic Anomalies. Interpretation Theory in Applied Geophys, 340-350.

[2] Liu, X.Z. (1993) The Principle and Application of Heavy Magnetic Anomaly Spectrum Analysis. Chongqing Press House, Chongqing, 132-167.

[3] Yan, Y.P., Dai, Q.W., Cai, J.X. and Lu, G.Y. (2011) Research on the Inversion Interpretation of Magnetic Abnormal Conjugate Element. Journal of Central South University (Natural Science Edition), 42, 731-737.

[4] Cai, J.X. (2019) Surface Magnetic Anomaly Triangulation Inversion. InternationalJournal of Geosciences, 10, 2156-2168. https://doi.org/10.4236/ijg.2019.102010

[5] Cai, J.X. (1989) Department of Magnetic Abnormal Triangle Interpretation. Geological Press, Beijing, 12-21.

[6] Cai, J.X., Li, P. and Dai, Q.W. (2016) Magnetic Abnormal Field Source Morphology Research. China Science and Technology Paper Online. http://www.paper.edu.cn/releasepaper/content/201605-596

[7] Yao, C.L. and Guan, J.N. (1997) Comprehensively Using the Total Field Anomalies and Its Vertical Gradient. Modern Geology, 78-83.

[8] Hou, C.C., Cai, Z.X. and Liu, K.J. (1985) Starting to Establish the Bit Field Conversion Interpretation System on the Curved Surface. Geophysics Journal, 28, 76-84.

[9] Yao, C.L. and Huang, W.N. (1997) Combined the Rapid Spline Flattening Method of Using Sites and Their Vertical Gradients. Geophysical Journal, 32, 229-236.

[10] An, Y.L., Chai, Y.P., Zhang, M.H., Jin, J.M. and Qiao, J.H. (2013) Quyang Ping with the Best Equivalent Source Model and Its Unit Bit Field Expression Derived New Method. Chinese Journal of Geophysics, 56, 2473-2483.

[11] Liu, J.L. and Wang, W.Y. (2007) In Changchun, Interested in Study. Geophysics, 50, 1351-1357.

[12] Cai, J.X. (2018) Differential Integrals of Curves and Inversion of Magnetic Source Morphology. Journal of Applied Mathematics and Physics, 6, 1160-1169.

https://doi.org/10.4236/jamp.2018.66098

[13] Cai, J.X. and Zhao, S.Q. (2017) Book the Interpretation of Blackboard Magnetic Anomaly under Undulating Terrain Conditions. China Science and Technology Paper Online Boutique Paper, 10, 1416-1424.

[14] Cai, J.X. (1994) Magnetic Triad. Zhongnan Institute of Mining and Metallurgical Technology, 25, 631-632.

[15] Cai, J.X. (1990) A Method of Magnetic Anomaly Decomposition Inversion with Distortion Correction. In: Computational Geophysical Research Collection, Earthquake Press, Beijing, 279-288.

[16] Yan, Y.P., Cai, J.X. and Dai, X.W. (2014) Theory and Methods of Magnetic Abnormal Conjugate Interpretation. Geological Press, Beijing, 89-91. 